\title{
Sufficient and necessary conditions for oscillation of linear fractional-order delay differential equations
}

\author{
Qiong Meng ${ }^{1 *}$, Zhen $\mathrm{Jin}^{2}$ and Guirong Liu'
}

\author{
"Correspondence: \\ mengqiong@qq.com \\ ${ }^{1}$ School of Mathematical Sciences, \\ Shanxi University, Taiyuan 030006, \\ Shanxi, China \\ Full list of author information is \\ available at the end of the article
}

\begin{abstract}
This paper studies the linear fractional-order delay differential equation

$$
{ }^{c} D_{-}^{\alpha} x(t)-p x(t-\tau)=0
$$

where $0<\alpha=\frac{\text { odd integer }}{\text { odd integer }}<1, p, \tau>0,{ }^{C} D_{-}^{\alpha} X(t)=-\Gamma^{-1}(1-\alpha) \int_{t}^{\infty}(s-t)^{-\alpha} x^{\prime}(s) d s$. We obtain the conclusion that

$$
p^{1 / \alpha} \tau>\alpha / e
$$

is a sufficient and necessary condition of the oscillations for all solutions of Eq. $\left(^{*}\right)$. At the same time, some sufficient conditions are obtained for the oscillations of multiple delays linear fractional differential equation. Several examples are given to illustrate our theorems.
\end{abstract}

MSC: $34 \mathrm{~K} 05 ; 34 \mathrm{~K} 37$

Keywords: Oscillation; Fractional differential equation; Autonomous; Delay; Linear

\section{Introduction}

In the past 30 years, fractional calculus has been developing rapidly in applications and theory. Fractional derivative has been used in many disciplines because of its nonlocal characteristics. A large number of fractional-order examples have also appeared in the fields of fluid mechanics, viscoelasticity, anomalous diffusion, control system, electrical engineering, electrochemistry, biology, etc. Many monographs [1-5] have been published. Many important research results have been obtained for the initial value problem, stability, attractiveness, boundary value problem, bifurcation, and diffusion of solutions of fractional differential equations. But compared with integer-order differential equations, there are still many questions to be studied, especially in the oscillation theory.

The research on the oscillation theory of functional differential equations has been developed during the past 40 years. There have been a lot of research results and many monographs, see [6-9]. These monographs provide very good results and methods. But for the

(c) The Author(s) 2021. This article is licensed under a Creative Commons Attribution 4.0 International License, which permits use, sharing, adaptation, distribution and reproduction in any medium or format, as long as you give appropriate credit to the original author(s) and the source, provide a link to the Creative Commons licence, and indicate if changes were made. The images or other third party material in this article are included in the article's Creative Commons licence, unless indicated otherwise in a credit line to the material. If material is not included in the article's Creative Commons licence and your intended use is not permitted by statutory regulation or exceeds the permitted use, you will need to obtain permission directly from the copyright holder. To view a copy of this licence, visit http://creativecommons.org/licenses/by/4.0/. 
fractional differential equations, there are few theoretical studies on the oscillation behavior, see [10-17]. This is because the functions of fractional derivatives lack good characteristics of the functions of integer-order derivative, such as monotonicity, concavity and convexity, etc. Now the oscillation theory of fractional differential equations is still in the development stage. We want to do some research work on the oscillation behavior for fractional differential equation.

In [11], the authors studied the fractional-order delay differential equations

$$
\dot{x}(t)+p D_{-}^{\alpha} x(t)+q x(t-\tau)=0,
$$

where $0<\alpha<1, p, q, \tau>0, D_{-}^{\alpha}$ is the Liouville fractional derivative on the half-axis $\mathbb{R}^{+}$ which is defined by Definition 2.1(2) in Sect. 2. They obtained the conclusion that every solution of Eq. ${ }^{(* *)}$ oscillates if and only if the characteristic equation

$$
\lambda+p(-\lambda)^{\alpha}+q e^{-\lambda \tau}=0
$$

has no negative real roots.

In this paper, we study the linear autonomous fractional-order delay differential equation

$$
{ }^{C} D_{-}^{\alpha} x(t)-p x(t-\tau)=0,
$$

where $0<\alpha=\frac{\text { odd integer }}{\text { odd integer }}<1, p, \tau>0,{ }^{C} D_{-}^{\alpha}$ is Caputo fractional derivative on the half-axis $\mathbb{R}^{+}$which is defined by Definition 2.1(3) in Sect. 2 .

In [6-8], the oscillation of the first-differential equation with delay

$$
x^{\prime}(t)+p x(t-\tau)=0, \quad p, \tau \in \mathbb{R},
$$

was studied. Their conclusion was that

$$
p \tau>1 / e
$$

is a necessary and sufficient condition for the oscillations for all solutions of Eq. (1.2).

In this paper, we obtain the following theorem.

Theorem 1.1 Assume that Condition $(H)$ in Sect. 3 holds. Then the following statements are equivalent:

(a) Every solution of Eq. (1.1) oscillates,

(b) $p^{1 / \alpha} \tau>\alpha / e$.

The proof of Theorem 1.1 is given in Sect. 3 .

The framework of this paper is as follows. In Sect. 2, we will provide some knowledge about fractional derivative and integral on the half-axis $\mathbb{R}^{+}$. In Sect. 3 , we obtain some sufficient and necessary conditions for oscillations of linear autonomous fractional-order delay differential equations via Laplace transform. At the same time, some sufficient conditions are obtained for the oscillations of multiple delay linear fractional differential equations. In Sect. 4, several examples are given to illustrate our theorems. 
Definition 1.1 ([6-8]) A nontrivial solution of a differential equation is said to be oscillatory if it has arbitrarily large zeros. Otherwise, it is called nonoscillatory. If a solution of a differential equation is nonoscillatory, it must be eventually positive or eventually negative.

\section{Preliminaries}

We will now provide some knowledge about fractional derivative and integral on the halfaxis $\mathbb{R}^{+}$, see [1-5].

Definition 2.1 ([4]) Let $\alpha \in(0,1)$.

(1) Liouville fractional integral for a function $f$ is defined by

$$
I_{-}^{\alpha} f(t)=\frac{1}{\Gamma(\alpha)} \int_{t}^{\infty} \frac{f(s)}{(s-t)^{1-\alpha}} d s, \quad t \in \mathbb{R}^{+}
$$

where $\Gamma(\cdot)>0$ is the gamma function, that is, $\Gamma(\alpha)=\int_{0}^{\infty} t^{\alpha-1} e^{-t} d t$.

(2) Liouville fractional derivative for a function $f$ is defined by

$$
D_{-}^{\alpha} f(t)=-\frac{1}{\Gamma(1-\alpha)} \frac{d}{d t} \int_{t}^{\infty} \frac{f(s)}{(s-t)^{\alpha}} d s, \quad t \in \mathbb{R}^{+} .
$$

(3) Caputo fractional derivative for a function $f$ is defined by

$$
{ }^{C} D_{-}^{\alpha} f(t)=-\frac{1}{\Gamma(1-\alpha)} \int_{t}^{\infty} \frac{f^{\prime}(s)}{(s-t)^{\alpha}} d s, \quad t \in \mathbb{R}^{+} .
$$

Remark 2.1 In [4, p. 83], Liouville fractional integral $I_{-}^{\alpha} f(t)$ and Liouville fractional derivative $D_{-}^{\alpha} f(t)$ exist for "sufficient good" functions $f(t)$; for example, for functions $f(t)$ in the space $C_{0}^{\infty}\left(\mathbb{R}^{+}\right)$of all infinitely differentiable functions on $\mathbb{R}^{+}$with a compact support.

Remark 2.2 ${ }^{C} D_{-}^{\alpha} f(t)=D_{-}^{\alpha} f(t), \alpha \in(0,1)$.

In fact,

$$
\begin{aligned}
{ }^{C} D_{-}^{\alpha} f(t) & =-\frac{1}{\Gamma(1-\alpha)} \int_{t}^{\infty} \frac{f^{\prime}(s)}{(s-t)^{\alpha}} d s=-\frac{1}{\Gamma(1-\alpha)} \int_{0}^{\infty} \frac{f^{\prime}(\xi+t)}{\xi^{\alpha}} d \xi, \\
D_{-}^{\alpha} f(t) & =-\frac{1}{\Gamma(1-\alpha)} \frac{d}{d t} \int_{t}^{\infty} \frac{f(s)}{(s-t)^{\alpha}} d s \\
& =-\frac{1}{\Gamma(1-\alpha)} \frac{d}{d t} \int_{0}^{\infty} \frac{f(\xi+t)}{\xi^{\alpha}} d \xi=-\frac{1}{\Gamma(1-\alpha)} \int_{0}^{\infty} \frac{f^{\prime}(\xi+t)}{\xi^{\alpha}} d \xi .
\end{aligned}
$$

Lemma 2.1 ([2, p. 242]; [4, p. 89]) Let $\alpha \in(0,1)$, then

$$
I_{-}^{\alpha} D_{-}^{\alpha} f(t)=D_{-}^{\alpha} I_{-}^{\alpha} f(t)=f(t),
$$

for all "sufficient good" functions $f(t)$. In particular, these formulas hold for $f(x) \in L_{1}\left(\mathbb{R}^{+}\right)$.

By Remark 2.2 and Lemma 2.1, we obtain the following lemma. 
Lemma 2.2 Let $\alpha \in(0,1)$, then

$$
I_{-}^{\alpha C} D_{-}^{\alpha} f(t)={ }^{C} D_{-}^{\alpha} I_{-}^{\alpha} f(t)=f(t) .
$$

Lemma $2.3\left(\left[4\right.\right.$, p. 98]) Let $\alpha \in(0,1), \operatorname{Re}(\lambda)<0$, then ${ }^{C} D_{-}^{\alpha} e^{\lambda t}=(-\lambda)^{\alpha} e^{\lambda t}$.

Lemma 2.4 ([2, p. 248]) Let $\alpha \in(0,1), \beta>0$, then

$$
D_{-}^{\alpha} \cos \beta t=\beta^{\alpha} \cos \left(\beta t-\frac{\alpha}{2} \pi\right), \quad D_{-}^{\alpha} \sin \beta t=\beta^{\alpha} \sin \left(\beta t-\frac{\alpha}{2} \pi\right) .
$$

By Remark 2.2 and Lemma 2.4, we obtain the following lemma.

Lemma 2.5 Let $\alpha \in(0,1), \beta>0$, then

$$
{ }^{C} D_{-}^{\alpha} \cos \beta t=\beta^{\alpha} \cos \left(\beta t-\frac{\alpha}{2} \pi\right), \quad{ }^{C} D_{-}^{\alpha} \sin \beta t=\beta^{\alpha} \sin \left(\beta t-\frac{\alpha}{2} \pi\right) .
$$

\section{Main theorems}

Consider the linear autonomous fractional-order delay differential equation

$$
{ }^{C} D_{-}^{\alpha} x(t)-\sum_{i=1}^{m} p_{i} x\left(t-\tau_{i}\right)=0
$$

where $0<\alpha=\frac{\text { odd integer }}{\text { odd integer }}<1, p_{i}, \tau_{i} \geq 0$ for $i=1,2, \ldots, m$.

In this paper, we need the following Condition $(\mathrm{H})$ :

$(H)$ The solutions for Eqs. (1.1) and (3.1) are exponentially bounded, that is, there exist positive constants $M$ and $\beta$ such that

$$
|x(t)| \leq M e^{\beta t}, \quad \text { for } t \geq 0
$$

Remark 3.1 The functions $e^{-t}$ and $\sin t$ are exponentially bounded.

Substituting function $x(t)=e^{s t}(s<0)$ into Eq. (3.1), we get

$$
(-s)^{\alpha}-\sum_{i=1}^{m} p_{i} e^{-s \tau_{i}}=0, \quad s<0
$$

Since $\alpha=\frac{\text { odd integer }}{\text { odd integer }}$, we obtain that the characteristic equation of Eq. (3.1) is

$$
s^{\alpha}+\sum_{i=1}^{m} p_{i} e^{-s \tau_{i}}=0, \quad s<0
$$

Theorem 3.1 Assume that Condition $(H)$ holds. Then the following statements are equivalent:

(a) Every solution of Eq. (3.1) oscillates;

(b) Equation (3.2) has no negative real roots. 
Proof (a) $\Rightarrow$ (b) It is obvious that if Eq. (3.2) has a negative real root $s_{0}$, then $e^{s_{0} t}$ is a nonoscillatory solution of Eq. (3.1).

(b) $\Rightarrow$ (a) Assume, on the contrary, that Eq. (3.1) has a positive solution $x(t)$. By Condition $(H)$, the Laplace transform $X(s)$ of $x(t)$ exists and is given by

$$
X(s)=\mathcal{L}(x)(s)=\int_{0}^{\infty} e^{-s t} x(t) d t
$$

First, by taking the Laplace transform for ${ }^{C} D_{-}^{\alpha} x(t)$, we get

$$
\begin{aligned}
\mathcal{L} & \left({ }^{C} D_{-}^{\alpha} x\right)(s) \\
& =-\frac{1}{\Gamma(1-\alpha)} \int_{0}^{\infty} e^{-s t}\left(\int_{t}^{\infty} \frac{x^{\prime}(u)}{(u-t)^{\alpha}} d u\right) d t \\
& =-\frac{1}{\Gamma(1-\alpha)} \int_{0}^{\infty} x^{\prime}(u)\left(\int_{0}^{u} e^{-s t}(u-t)^{-\alpha} d t\right) d u \\
& =\frac{1}{\Gamma(1-\alpha)} \int_{0}^{\infty} x^{\prime}(u) e^{-s u}\left(\int_{u}^{0} e^{s v} v^{-\alpha} d v\right) d u \quad(t=u-v) \\
& =\frac{1}{s^{1-\alpha} \Gamma(1-\alpha)} \int_{0}^{\infty} x^{\prime}(u) e^{-s u}\left(\int_{-s u}^{0} e^{-z} z^{-\alpha} d z\right) d u \quad(z=-s v) \\
& =-s^{\alpha-1} \int_{0}^{\infty} x^{\prime}(u) e^{-s u}\left(\frac{\int_{0}^{-s u} e^{-z} z^{-\alpha} d z}{\int_{0}^{\infty} e^{-z} z^{-\alpha} d z}\right) d u \quad\left(\Gamma(1-\alpha)=\int_{0}^{\infty} e^{-z} z^{(1-\alpha)-1} d z\right) \\
& =-A s^{\alpha-1} \int_{0}^{\infty} x^{\prime}(u) e^{-s u} d u\left(0<A=\frac{\int_{0}^{-s \xi} e^{-z} z^{-\alpha} d z}{\int_{0}^{\infty} e^{-z} z^{-\alpha} d z}<1,0 \leq \xi \leq \infty\right) \\
& =-A s^{\alpha-1}\left(\left[x(u) e^{-s u}\right]_{0}^{\infty}+s \int_{0}^{\infty} x(u) e^{-s u} d u\right) \\
& =-A s^{\alpha-1}\left(x(0)+s \int_{0}^{\infty} x(u) e^{-s u} d u\right)=-A s^{\alpha-1} x(0)-A s^{\alpha} X(s),
\end{aligned}
$$

where $A$ exists by Lemma 2.2 [11], the mean value theorem for the infinite interval.

Now, taking the Laplace transforms on both sides of Eq. (3.1), we get

$$
-A s^{\alpha} X(s)-A s^{\alpha-1} x(0)-\sum_{i=1}^{m} p_{i} \int_{0}^{\infty} e^{-s t} x\left(t-\tau_{i}\right) d t=0,
$$

that is,

$$
A s^{\alpha} X(s)+A s^{\alpha-1} x(0)+\sum_{i=1}^{m} p_{i} e^{-s \tau_{i}} X(s)+\sum_{i=1}^{m} p_{i} e^{-s \tau_{i}} \int_{-\tau_{i}}^{0} e^{-s t} x(t) d t=0
$$

or

$$
\left(A s^{\alpha}+\sum_{i=1}^{m} p_{i} e^{-s \tau_{i}}\right) X(s)=-A s^{\alpha-1} x(0)-\sum_{i=1}^{m} p_{i} e^{-s \tau_{i}} X(s) \int_{-\tau_{i}}^{0} e^{-s t} x(t) d t .
$$

Then

$$
H(s) X(s)=\Phi(s),
$$


where

$$
H(s)=A s^{\alpha}+\sum_{i=1}^{m} p_{i} e^{-s \tau_{i}}, \quad \Phi(s)=-A s^{\alpha-1} x(0)-\sum_{i=1}^{m} p_{i} e^{-s \tau_{i}} X(s) \int_{-\tau_{i}}^{0} e^{-s t} x(t) d t .
$$

Let

$$
F(s)=s^{\alpha}+\sum_{i=1}^{m} p_{i} e^{-s \tau_{i}}
$$

Since $F(0)>0, F(s)>0$ for $s<0$ from condition $(b)$. Hence $H(s)>F(s)>0$ for $s<0$. As $s \rightarrow-\infty$, we get a contradiction in (3.5), since $H(s) X(s)>0$, but $\Phi(s)<0$.

Proof of Theorem 1.1 The characteristic equation of Eq. (1.1) is

$$
F(s)=s^{\alpha}+p e^{-s \tau}=0, \quad s<0 .
$$

By Theorem 3.1, we need to prove that

$$
p^{1 / \alpha} \tau>\alpha / e \Leftrightarrow F(s)>0, \quad \text { for } s<0 .
$$

From (3.6), we have

$$
F(s)=(-s)^{\alpha}\left[-1+\frac{p e^{-s \tau}}{(-s)^{\alpha}}\right]
$$

Let

$$
h(\lambda)=-1+\frac{p e^{\lambda \tau}}{\lambda^{\alpha}}, \quad \text { for } \lambda>0 .
$$

Then

$$
h(\lambda) \geq \min _{\lambda>0} h(\lambda)=h\left(\frac{\alpha}{\tau}\right)=-1+p \tau^{\alpha}\left(\frac{e}{\alpha}\right)^{\alpha} .
$$

So, we get

$$
p^{1 / \alpha} \tau>\alpha / e \Leftrightarrow p \tau^{\alpha}>(\alpha / e)^{\alpha} \Leftrightarrow h(\lambda)>0, \quad \lambda>0 \quad \Leftrightarrow \quad F(s)>0, \quad s<0 .
$$

The proof of Theorem 1.1 is complete.

In the following, we obtain some sufficient conditions given explicitly in terms of the coefficients and the delays, for the oscillations of all solutions of Eq. (3.1).

Theorem 3.2 Assume that $p_{i}, \tau_{i} \geq 0$ for $i=1,2, \ldots, m$. Then each of the following two conditions is sufficient for the oscillation for all solutions of Eq. (3.1):

(a) $\sum_{i=1}^{m} p_{i} \tau_{i}^{\alpha}>\left(\frac{\alpha}{e}\right)^{\alpha}$;

(b) $\prod_{i=1}^{m} p_{i}^{1 / m} \tau_{i}^{\alpha / m}>\left(\frac{\alpha}{e}\right)^{\alpha}$. 
Proof (a) For $s<0$,

$$
s^{\alpha}+\sum_{i=1}^{m} p_{i} e^{-s \tau_{i}}=(-s)^{\alpha}\left[-1+\sum_{i=1}^{m} p_{i} \frac{e^{-s \tau_{i}}}{(-s)^{\alpha}}\right] \geq(-s)^{\alpha}\left[-1+\left(\frac{e}{\alpha}\right)^{\alpha} \sum_{i=1}^{m} p_{i} \tau_{i}^{\alpha}\right]>0
$$

which shows that Eq. (3.2) has no negative real roots. So $F(s)>0$ for $s<0$, the result follows as a consequence of Theorem 3.1.

(b) By using the arithmetic-geometric mean inequality

$$
\left(\prod_{i=1}^{m} p_{i}\right)^{1 / m} \leq \frac{1}{m} \sum_{i=1}^{m} p_{i}
$$

we find that for $s<0$,

$$
\begin{aligned}
s^{\alpha}+\sum_{i=1}^{m} p_{i} e^{-s \tau_{i}} & \geq s^{\alpha}+m\left(\prod_{i=1}^{m} p_{i} e^{-s \tau_{i}}\right)^{1 / m} \\
& =(-s)^{\alpha}\left(-1+\prod_{i=1}^{m} p_{i}^{1 / m} \frac{e^{-s \tau_{i} / m}}{(-s)^{\alpha / m}}\right) \\
& \geq(-s)^{\alpha}\left(-1+\left(\frac{e}{\alpha}\right)^{\alpha} \prod_{i=1}^{m} p_{i}^{1 / m} \tau_{i}^{\alpha / m}\right)>0,
\end{aligned}
$$

which shows that Eq. (3.2) has no negative real roots. So $F(s)>0$ for $s<0$, the result follows as a consequence of Theorem 3.1. The proof Theorem 3.2 is complete.

Theorem 3.3 Assume that $p_{i}, \tau_{i} \geq 0$ for $i=1,2, \ldots, m$.

(1) If

$$
\left(\sum_{i=1}^{m} p_{i}\right)\left(\max _{1 \leq i \leq m} \tau_{i}^{\alpha}\right) \leq\left(\frac{\alpha}{e}\right)^{\alpha}
$$

holds, then Eq. (3.1) has a nonoscillatory solution.

(2) If

$$
\left(\sum_{i=1}^{m} p_{i}\right)\left(\min _{1 \leq i \leq m} \tau_{i}^{\alpha}\right)>\left(\frac{\alpha}{e}\right)^{\alpha}
$$

holds, then every solution of Eq. (3.1) oscillates.

Proof (1) Let

$$
\tau=\max _{1 \leq i \leq m} \tau_{i}, \quad F(s)=s^{\alpha}+\sum_{i=1}^{m} p_{i} e^{-s \tau_{i}}
$$

If $\tau=0, F(s)=0$ has a real $\operatorname{root} s=-\left(\sum_{i=1}^{m} p_{i}\right)^{1 / \alpha}$. 
If $\tau \neq 0$, we have

$$
\begin{aligned}
F(0) F\left(-\frac{\alpha}{\tau}\right) & =\left(\sum_{i=1}^{m} p_{i}\right)\left[-\left(\frac{\alpha}{\tau}\right)^{\alpha}+\sum_{i=1}^{m} p_{i} e^{\alpha \tau_{i} / \tau}\right] \\
& \leq\left(\sum_{i=1}^{m} p_{i}\right)\left[-\left(\frac{\alpha}{\tau}\right)^{\alpha}+\sum_{i=1}^{m} p_{i} e^{\alpha}\right] \\
& =\left(\frac{e}{\tau}\right)^{\alpha}\left(\sum_{i=1}^{m} p_{i}\right)\left[-\left(\frac{\alpha}{e}\right)^{\alpha}+\sum_{i=1}^{m} p_{i} \tau^{\alpha}\right] \leq 0,
\end{aligned}
$$

which shows that Eq. (3.2) has a negative real root $s_{0} \in[-\alpha / \tau, 0)$. Then $e^{s_{0} t}$ is a nonoscillatory solution of Eq. (3.1).

(2) The fact that (3.10) is a sufficient condition for all solutions of Eq. (3.1) to oscillate follows immediately as a corollary of condition (a) of Theorem 3.1. The proof Theorem 3.3 is complete.

\section{Examples}

Example 4.1 Consider the fractional differential equation without delay

$$
{ }^{C} D_{-}^{\alpha} x(t)-p x(t)=0
$$

where $0<\alpha=\frac{\text { odd integer }}{\text { odd integer }}<1, p>0$. Then $x(t)=e^{-p^{1 / \alpha} t}$ is a solution of Eq. (4.1).

Example 4.2 Consider the fractional-order delay differential equation

$$
{ }^{C} D_{-}^{\alpha} x(t)-\left(\frac{\alpha}{e}\right)^{\alpha} x(t-1)=0
$$

where $0<\alpha=\frac{\text { odd integer }}{\text { odd integer }}<1, p=(\alpha / e)^{\alpha}, \tau=1$. Since $p^{1 / \alpha} \tau=\alpha / e$, by Theorem 1.1, Eq. (4.2) has nonoscillatory solutions. In fact, $x(t)=e^{-\alpha t}$ is a nonoscillatory solution of Eq. (4.2).

Example 4.3 Consider the fractional-order delay differential equation

$$
{ }^{C} D_{-}^{\alpha} x(t)-x\left(t-\frac{\alpha \pi}{2}\right)=0,
$$

where $0<\alpha=\frac{\text { odd integer }}{\text { odd integer }}<1, p=1, \tau=\alpha \pi / 2$. Since $p^{1 / \alpha} \tau>\alpha / e$, by Theorem 1.1, every solution of Eq. (4.3) oscillates. In fact, $x(t)=\sin t$ is an oscillatory solution of Eq. (4.3).

\section{Acknowledgements}

The authors thank the anonymous referees for their valuable suggestions and comments.

\section{Funding}

Research supported by Shanxi Key Laboratory of Mathematical Techniques and Big Data Analysis on Disease Control and Prevention (No. 201705D111006), National Natural Science Foundation of China (No. A011403) and Shanxi Natural Science Foundation (No. 201901D111009). 
Availability of data and materials

Not applicable.

Ethics approval and consent to participate

Not applicable.

Competing interests

The authors declare that they have no competing interests.

Consent for publication

Not applicable.

\section{Authors' contributions}

All authors contributed equally to the writing of this paper. All authors read and approved the final manuscript.

\section{Author details}

'School of Mathematical Sciences, Shanxi University, Taiyuan 030006, Shanxi, China. ${ }^{2}$ Complex Systems Research Center, Shanxi University, Taiyuan 030006, Shanxi, China.

\section{Publisher's Note}

Springer Nature remains neutral with regard to jurisdictional claims in published maps and institutional affiliations.

Received: 25 April 2020 Accepted: 6 January 2021 Published online: 29 January 2021

\section{References}

1. Podlubny, I.: Fractional Differential Equations. Academic Press, San Diego (1999)

2. Miller, K.S., Ross, B.: An Introduction to the Fractional Calculus and Fractional Differential Equations. Wiley, New York (1993)

3. Smoko, S.G., Kilbas, A.A., Marichev, O.I.: Fractional Integrals and Derivatives: Theory and Application. Gordon \& Breach, New York (1993)

4. Kilbas, A.A., Srivastava, H.M., Trujillo, J.J.: Theory and Applications of Fractional Differential Equations. Elsevier, Amsterdam (2006)

5. Zhou, Y: Basic Theory of Fractional Differential Equations. World Scientific, Singapore (2014)

6. Györi, I., Ladas, G.: Oscillation Theory of Delay Differential Equations with Applications, Clarendon, Oxford (1991)

7. Gopalsamy, K.: Stability and Oscillation in Delay Differential Equations of Population Dynamics. Kluwer Academic Boston (1992)

8. Zheng, Z.: Theory of Functional Differential Equations. Anhui Education Press, China (1992) (in Chinese)

9. Kuang, Y.: Delay Differential Equations with Applications in Population Dynamics. Mathematics in Science and Engineering, vol. 191. Academic Press, San Diego (1993)

10. Bolat, Y.: On the oscillation of fractional-order delay differential equations with constant coefficients. Commun. Nonlinear Sci. Numer. Simul. 19, 3988-3993 (2014)

11. Wang, Y., Han, Z:: Comment on "On the oscillation of fractional-order delay differential equations with constant coefficients" [Commun. Nonlinear Sci. 19(11) (2014) 3988-3993]. Commun. Nonlinear Sci. Numer. Simul. 26, 195-200 (2015)

12. Prakash, P., Harikrishnan, S., Nieto, J.J., Kim, J.-H.: Oscillation of a time fractional partial differential equation. Electron. J. Qual. Theory Differ. Equ. 2014, 15, 1-10 (2014)

13. Grace, S., Agarwal, R., Wong, P., et al.: On the oscillation of fractional differential equations. Fract. Calc. Appl. Anal. 15 , 222-231 (2012)

14. Zhou, Y., Ahmad, B., Chen, F., Alsaedi, A.: Oscillation for fractional partial differential equations. Bull. Malays. Math. Soc. 42, 449-465 (2019)

15. Zhu, P., Xiang, Q.: Oscillation criteria for a class of fractional delay differential equations. Adv. Differ. Equ. 2018, 403 (2018)

16. Abdalla, B., Abdeljawad, T:: On the oscillation of Hadamard fractional differential equations. Adv. Differ. Equ. 2018, 409 (2018)

17. Alzabut, J.O., Abdeljawad, T:. Sufficient conditions for the oscillation of nonlinear fractional difference equations. J. Fract. Calc. Appl. 5(1), 177-187 (2014) 